\title{
Interpretation of Non-Genetic Oral and Maxillofacial Osteogenic Conditions in the Basis of New Findings in the Field of Osteoblastogenesis and Osteoclastogenesis
}

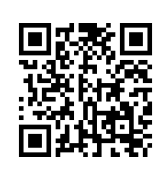

\author{
Yang $\mathrm{Gu}^{*}$ \\ Yang Gu, Assistant Professor and Oral Pathologist, Director of Oral Pathology Clinic, Department of Oral and Maxillofacial Sciences, Faculty of Dentistry, \\ Dalhousie University, Canada
}

Received: June 26, 2018; Published: July 03, 2018

*Corresponding author: Yang Gu, Assistant Professor and Oral Pathologist, Director of Oral Pathology Clinic, Department of Oral and Maxillofacial Sciences, Faculty of Dentistry, Dalhousie University, Canada

Abstract

Aim: The paper reorganized new findings in the field of osteoblastogenesis and osteoclastogenesis for clinicians to identify systemic and local risks of oral and maxillofacial osteogenic conditions (OMOCs) that are not related to genetic diseases.

Method: The non-systemic review was undertaken by searching pertinent key wards in English literature. Case report and publications with weak levels of evidence were excluded.

Results: Coupling factors from endocrine and paracrine systems affect osteoblastogenesis and osteoclastogenesis in different pathways. Levels of hormones change with age or by medical conditions. Cytokine accumulations are triggered by the local mechanical force or ischemia. Osteoblasts take a leading role in the maturation of osteoclasts, while the activity of osteoclasts could be an initial event in a local osteogenic lesion. The entangling relation between osteoblasts and osteoclasts derives some patterns for OMOCs radiographically.

Discussion: A triple-hit frame composited by timings, coupling factors from endocrine and paracrine systems is an approachable method to explore local and systemic risks for OMOCs.

Keywords: Osteoblast; Osteoclast; Hormone; Cytokine; Radiolucency; Radiopacity

Abbreviations: OPG: Osteoprotegerin; RANKL: Nuclear Factor-kB Ligand; GI: Gastrointestinal System; S1P: Sphingosine 1-Phosphate; TNF: Tumor Necrosis Factor; IL: Interleukin; FasL: Fas Ligand; PDGF: Platelet-Derived Growth Factor; VEGF: Vascular Endothelial Growth Factor

\section{Introduction}

The ratio of nuclear factor kB ligand (RANKL) to osteoprotegerin $(\mathrm{OPG})$ is not the only tool to explore the interaction between osteoblasts and osteoclasts. Sphingosine 1-phosphate (S1P) and Sclerostin work on osteoblasts in the opposite way. Eight hormones (Growth Hormone, Calcitriol, Androgen, Estrogen, Calcitonin, Thyroxin, Cortisol and Parathyroid Hormone) regulate behaviors of osteoblasts and osteoclasts at different levels. Seven cytokines (Macrophage colony-stimulating factor, Tumor necrosis factoralpha, Histamine, Interleukin-1, 4, 12 and 13) affect their behaviors in different pathways. Lifespans of osteoblasts, osteoclasts, fibroblasts, and endothelium are different, while hormone levels of Growth Hormone, Estrogen, Androgen and Thyroxin change with aging. The unbalanced behavior of osteoblasts and osteoclasts could induce the excessive bone formation and the abnormal bone resorption, which project on a radiography as radiolucency or radiopacity or mixed lucent-opaque lesions. The paper tried to reorganize new findings for clinicians to identify systemic and local risks of non-genetic oral and maxillofacial osteogenic conditions (OMOCs).

\section{Method}

The non-systematic review identified recent original research papers, systematic reviews, meta-analysis articles and narrative reviews from author input supplemented by the PubMed, Google Scholar and PLOS ONE. Keywords included osteoblasts, osteoclasts, hormone, cytokine, radiolucency, radiopacity and oral and maxillofacial osteogenic conditions. Case reports and publications with weak levels of evidence were excluded. 


\section{Results}

\section{Osteoblast and Osteoclast}

Osteoblasts differentiate from mesenchymal osteoprogenitors who are governed by the RUNX2/CBFA1 transcription factor network and the WNT/ beta-catenin signal pathway [1]. Osteoblasts synthesize, transport and arrange proteins of bone matrix and initiate the process of mineralization. The osteoblast will transform into an osteocyte when it is surrounded by newly deposited bone matrix. Common markers for active osteoblasts are Alkaline Phosphatase (ALP) and Osteocalcin [2,3]. Osteoclast precursors differentiate from hematopoietic progenitor cells that are probably governed by the ERK-Akt pathway through c-Fms and c-Kit receptors [4]. An osteoclast precursor (mononuclear osteoclast) will transform into a mature osteoclast (multinuclear osteoclast) when the NF-kB signaling and the tyrosine kinase receptor are activated. Multinuclear osteoclasts digest proteins of bone matrix and initiate the process of demineralization. Common markers for mature osteoclasts are Tartrate-Resistant Acid Phosphatase (TRAP) and Cathepsin K [2,5].

\section{Interaction Between Osteoblasts and Osteoclasts (Illustration 1)}

An osteoblast presents a receptor activator of nuclear factor-kB ligand (RANKL) and Macrophage colony-stimulating factor (M-CSF) on the surface, while a mononuclear osteoclast expresses RANKL receptor (RANK) and M-CSF receptor (a type of tyrosine kinase receptors). When they bind together the NF-kB signaling and the tyrosine kinase receptor are activated. A multinuclear osteoclast matures1. Osteoblasts take a leading role in the maturation of osteoclasts.

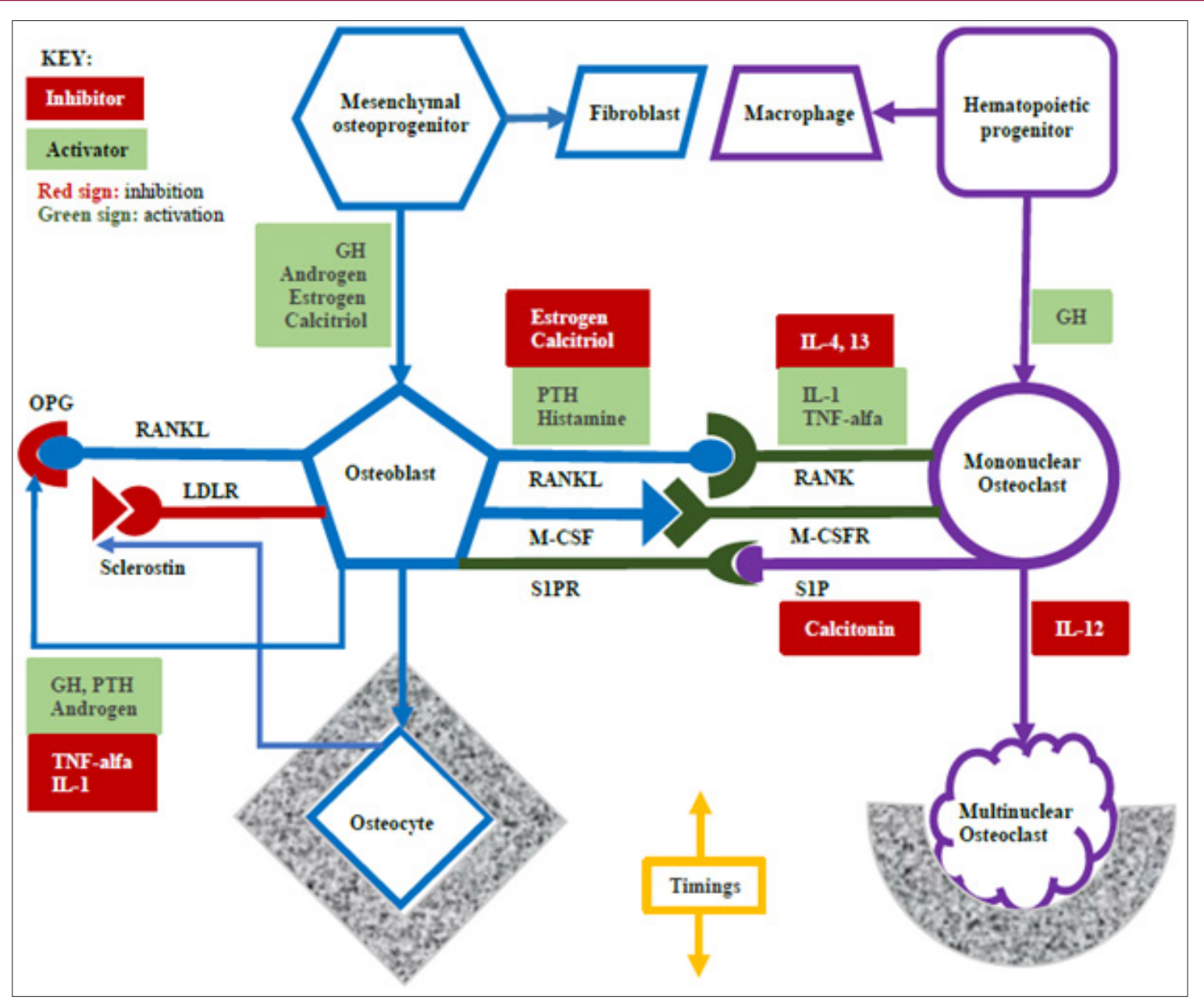

Illustration 1: Triple-hit frame: Interactions between osteoblasts and osteoclasts are regulated by coupling factors from endocrine and paracrine systems (in color). GH: growth hormone; IL: interleukin; PTH: parathyroid hormone; TNF: tumor necrosis factor; OPG: osteoprotegerin; RANKL: nuclear factor-kB ligand; RANK: RANKL receptor; LDLR: low-density lipoprotein receptor; M-CSF: macrophage colony- stimulating factor; M-CSFR: M-CSF receptor; S1P: Sphingosine 1-phosphate; S1PR: S1P receptor.

An osteoblast also produces osteoprotegerin (OPG), which is a "decoy" receptor binding RANKL. It blocks RANKL to bind RANK [6]. It is a negative control to the maturation of osteoclasts. An osteoclast produces Sphingosine 1-phosphate (S1P), which binds the S1P receptor of an osteoblast [7]. The signal promotes osteoblasts to produce bone matrix. It is a positive control to the function of osteoblasts. However, an osteocyte produces Sclerostin to bind the low-density lipoprotein receptor (LDLR)-related protein of an osteoblast [8]. It inhibits osteoblasts to produce bone matrix. It is a negative control to the function of osteoblasts. 
RANKL and OPG, both from osteoblasts, oppose one another. S1P and Sclerostin work on osteoblasts but in the opposite way. Who controls the direction? They are endocrine molecules (e.g. hormones) and paracrine molecules (e.g. cytokines).

\section{Hormones (Endocrine Molecules) (Table 1) and (Illustration 1)}

\section{Bone Formation as a Priority:}

a) Growth hormone (GH) binds $\mathrm{GH}$ receptors (GHR) of osteoblasts and osteoclast precursors to promote their differentiation but also stimulates osteoblasts to produce OPG resulting in the bone formation as a priority [9].

b) Estrogen binds estrogen receptors (ER) of osteoprogenitors to promote the differentiation of osteoblasts and binds ERs of osteoblasts to inhibit osteoblasts to present RANKL resulting in the bone formation as a priority $[10,11]$.

c) Androgen binds an androgen receptor (AR) of osteoprogenitors to promote the differentiation of osteoblasts and binds ERs to stimulate osteoblasts to produce OPG resulting in the bone formation as a priority $[12,13]$.

\section{Mineral Homeostasis as a Priority:}

a) Parathyroid hormone (PTH) binds PTH receptors of osteoblasts to increase the expression of RANKL and to decrease the production of $\mathrm{OPG}(\mathrm{E} / \mathrm{C})$ resulting in the maturation of osteoclasts and following with the bone resorption as a priority, but another function is activating alpha-hydroxylase to promote Calcitriol formation. Hypocalcemia is the stimulator of PTH [14].

b) Calcitriol binds Vitamin $\mathrm{D}$ receptors (VDR) of osteoprogenitors to promote the differentiation of osteoblasts [15], also works as a suppressor of inhibiting osteoblasts to express RANKL [16] resulting in the bone formation as a priority, but the well-known function is helping the intestine and kidneys to absorb calcium. Hypocalcemia and PTH are stimulators of Calcitriol.

c) Calcitonin binds a Calcitonin receptor (CTR) of a mature osteoclast to inhibit the production of Sphingosine 1 phosphate (S1P) $[7,17]$. However, the S1P binds an S1P receptor of an osteoblast to promote the production of bone matrix. Hypercalcemia is the stimulator of Calcitonin.

\section{Negative Effect on Bone:}

a) Thyroxin binds thyroid hormone receptors (THR) of osteoblasts and osteoclasts to promote their functions, but it shortens the bone remodeling cycle resulting in a loss of about $10 \%$ bone mass per cycle [18].

b) Cortisol inhibits osteoclastogenesis and osteoblastogenesis by the FOXO antioxidant pathway and binds the Glucocorticosteroid receptor (GR) of endothelium to inhibit angiogenesis resulting in the osteoporosis $[19,20]$.

Hormones control the behavior of osteoblasts and osteoclasts through specific receptors in different directions. The natural course of bone formation and bone resorption is changed by the imbalance of hormone levels. Aetiologies of the abnormal secretion of hormones are complicated. For example, hypocalcemia usually is associated with Gastrointestinal conditions and renal dysfunctions, while hypercalcemia usually is related to renal failure and systemic granulomatous diseases. Practically, the systemic risks of OMOCs could be identified from the medical history and the laboratory examination.

\section{Paracrine Molecules (Table 1) and (Illustration 1)}

Stromal cells (including endothelium and fibroblast) and inflammatory cells produce cytokines when facing ischemia and inflammation.

Table 1: Pathways of hormones and cytokines working on osteoblasts and osteoclasts and alterations caused by common medical conditions and drugs.

\begin{tabular}{|c|c|c|c|c|c|}
\hline & RANKL: OPG Ratio & Osteoblast & Osteoclast & Another Pathway & $\begin{array}{c}\text { Medical Conditions / } \\
\text { Drugs }\end{array}$ \\
\hline $\begin{array}{c}\text { Growth } \\
\text { Hormone9 }\end{array}$ & Decrease (high OPG) & $\begin{array}{c}\text { Promote } \\
\text { differentiation }\end{array}$ & $\begin{array}{c}\text { Promote } \\
\text { differentiation }\end{array}$ & $\mathrm{N} / \mathrm{A}$ & $\begin{array}{l}\text { Decline in aging; } \\
\text { Acromegaly }\end{array}$ \\
\hline Calcitriol15,16 & Decrease (low RANKL) & $\begin{array}{c}\text { Promote } \\
\text { differentiation }\end{array}$ & N/A & N/A & $\begin{array}{l}\text { Hypocalcemia due to } \\
\text { GI or renal conditions }\end{array}$ \\
\hline Estrogen 10,11 & Decrease (low RANKL) & $\begin{array}{c}\text { Promote } \\
\text { differentiation }\end{array}$ & $\mathrm{N} / \mathrm{A}$ & $\mathrm{N} / \mathrm{A}$ & $\begin{array}{l}\text { Decline in aging; } \\
\text { Female steroid drug }\end{array}$ \\
\hline Androgen12,13 & Decrease (high OPG) & $\begin{array}{c}\text { Promote } \\
\text { differentiation }\end{array}$ & N/A & N/A & $\begin{array}{l}\text { Decline in aging; Male } \\
\text { steroid drug }\end{array}$ \\
\hline $\begin{array}{l}\text { Parathyroid } \\
\text { hormone14 }\end{array}$ & $\begin{array}{c}\text { Increase (high RANKL; } \\
\text { low OPG) }\end{array}$ & N/A & N/A & $\mathrm{N} / \mathrm{A}$ & $\begin{array}{l}\text { Hyperparathyroidism } \\
\text { 1st,2nd and 3rd }\end{array}$ \\
\hline Thyroxin 18 & $\mathrm{~N} / \mathrm{A}$ & Promote function & Promote function & $\begin{array}{c}\text { Shorten remodeling } \\
\text { cycle }\end{array}$ & $\begin{array}{l}\text { Decline in aging; } \\
\text { Hyperthyroidism }\end{array}$ \\
\hline Cortisol19,20 & $\mathrm{N} / \mathrm{A}$ & $\begin{array}{c}\text { Inhibit } \\
\text { osteoblastogenesis }\end{array}$ & $\begin{array}{c}\text { Inhibit } \\
\text { osteoclastogenesis }\end{array}$ & Inhibit angiogenesis & $\begin{array}{l}\text { Hypercortisolism; } \\
\text { Corticosteroid drug }\end{array}$ \\
\hline Calcitonin7,17 & $\mathrm{N} / \mathrm{A}$ & $\mathrm{N} / \mathrm{A}$ & Inhibit S1P secretion & $\mathrm{N} / \mathrm{A}$ & $\begin{array}{c}\text { Hypercalcemia; } \\
\text { Hypercalcitoninemia }\end{array}$ \\
\hline
\end{tabular}




\begin{tabular}{|c|c|c|c|c|c|}
\hline TNF-alfa23 & Increase (low OPG) & N/A & $\begin{array}{c}\text { Increase RANK } \\
\text { expression }\end{array}$ & $\begin{array}{c}\text { An antagonist to treat } \\
\text { osteoporosis: } \\
\text { Denosumab }\end{array}$ & $\begin{array}{c}\text { An antagonist to treat } \\
\text { rheumatoid arthritis: } \\
\text { Ankinra }\end{array}$ \\
\hline IL-123 & Increase (low OPG) & N/A & $\begin{array}{c}\text { Increase RANK } \\
\text { expression }\end{array}$ & N/A \\
\hline Histamine24 & Increase (high RANKL) & N/A & N/A & N/A treatment: \\
\hline IL-4,1322 & N/A & N/A & $\begin{array}{c}\text { Decrease RANK } \\
\text { expression }\end{array}$ & $\begin{array}{c}\text { An antagonist to treat } \\
\text { Eczema: Pitrakinra }\end{array}$ \\
\hline IL-1221 & N/A & N/A & $\begin{array}{c}\text { Increase apoptosis by } \\
\text { FASL }\end{array}$ & $\begin{array}{c}\text { An antagonist to treat } \\
\text { Psoriasis: Ustekinumb }\end{array}$ \\
\hline PGDF25 & N/A & N/A & N/A & Promote angiogenesis & $\begin{array}{c}\text { An antagonist to treat } \\
\text { malignancy: Imatinib }\end{array}$ \\
\hline VEG F26 & N/A & N/A & N/A & Promote angiogenesis & $\begin{array}{c}\text { An antagonist to } \\
\text { treat malignancy: } \\
\text { Bevacizumab }\end{array}$ \\
\hline
\end{tabular}

Note: OPG: osteoprotegerin; RANKL: nuclear factor-kB ligand; GI: gastrointestinal system; S1P: Sphingosine 1-phosphate; TNF: tumor necrosis factor; IL: interleukin; FasL: Fas ligand; PDGF: Platelet-derived growth factor; VEGF: Vascular endothelial growth factor.

a) Inhibition of Osteoclastogenesis: Interleukin-12 (IL12) increase the FAS ligand expression to promote osteoclast apoptosis [21]. Interleukin-4 (IL-4) and 13 inhibit osteoclasts to express RANK [22].

b) Activation of Osteoclastogenesis: Tumor necrosis factoralfa (TNF-alfa) and Interleukin-1 (IL-1) promote osteoclasts to express RANK and inhibit osteoblasts to produce OPG [23]. Histamine promotes osteoblasts to produce RANKL [24].

c) Platelet-derived growth factor (PDGF) and Vascular endothelial growth factor (VEGF) work with endothelium to promote angiogenesis by the P13K-Akt pathway $[25,26]$.

Osteocytes and inflammatory cells produce cell signaling proteins when bearing physiological mechanical force.

a) Inflammatory cells produce TNF-alfa [27].

b) Osteocytes decrease the production of Sclerostin [28].

c) Estrogen promotes the appositional bone formation [15].

Paracrine molecules affect osteoblasts and osteoclasts through common receptors in different pathways. Those injurious stimuli, ischemia, inflammation and mechanical force, are common in the oral cavity. For example, a dental procedure usually is performed under a local anesthesia containing epinephrine that increases the risk of local ischemia, while occlusal force during chewing, biting or clenching is not uncommon in periodontal regions. Practically, local risks of oral and maxillofacial osteogenic lesions could be identified from the dental history and oral examinations.

\section{Timings}

When clinicians list significant findings of oral and maxillofacial osteogenic conditions the information about timings tells us when did it start? where was the initial problem? what should be linked to the systemic risk? and how did a local risk exacerbate the condition? Timing is the key to open a multifactorial program.

Lifespans of Osteoblasts, Osteoclasts and Fibroblasts: Bone formation or bone resorption is achieved by an osteogenic multicellular group (OMG) including osteocytes, osteoblasts, osteoclasts, fibroblasts, endothelium, mesenchymal and hematopoietic precursors $[1,29,30]$. The lifespan of osteoblasts is around three months, while the two-week cycle is for osteoclasts [31]. The lifespan of fibroblasts is around two months, but the half-life of osteocytes is around 25 years. Endothelium and precursors only survive several days. In the system osteoblasts and fibroblasts are cornerstones, while the most changeable cells are osteoclasts and endothelium. The balance of osteoblastogenesis and osteoclastogenesis is already programmed by coupling factors that work as activators or inhibitors from endocrine or paracrine systems (Illustration 1). Therefore, the system can be reprogramed on condition that unbalanced coupling factors consistently work with the brand-new OMG for 3-6 months.

Hormones and Aging (Charts 1 \& 2): Levels of PTH, Calcitriol, and Calcitonin basically change when hypocalcemia or hypercalcemia exists. The level of Cortisol changes in a day and is affected by stress, rather than aging. The level of Thyroxin usually remains stable and starts to decrease in age 60 and significantly drops at 70 years old (C) [32]. GH has a peak range between 15 y/o and $25 \mathrm{y} / \mathrm{o}$ and gradually declines with the time. Insulin-like growth factor (IGF) is the main mediator of GH. We check the level of IGF instead of GH clinically (C) [33]. Male and Female have Estrogen and bioavailable Testosterone (a type of androgen) both, but their levels change with aging in different ways. It is less likely to argue a conclusion that the normal osteoblastogenesis in women is gradually weakening after 45 years old if not enough Vitamin D is supplied. 


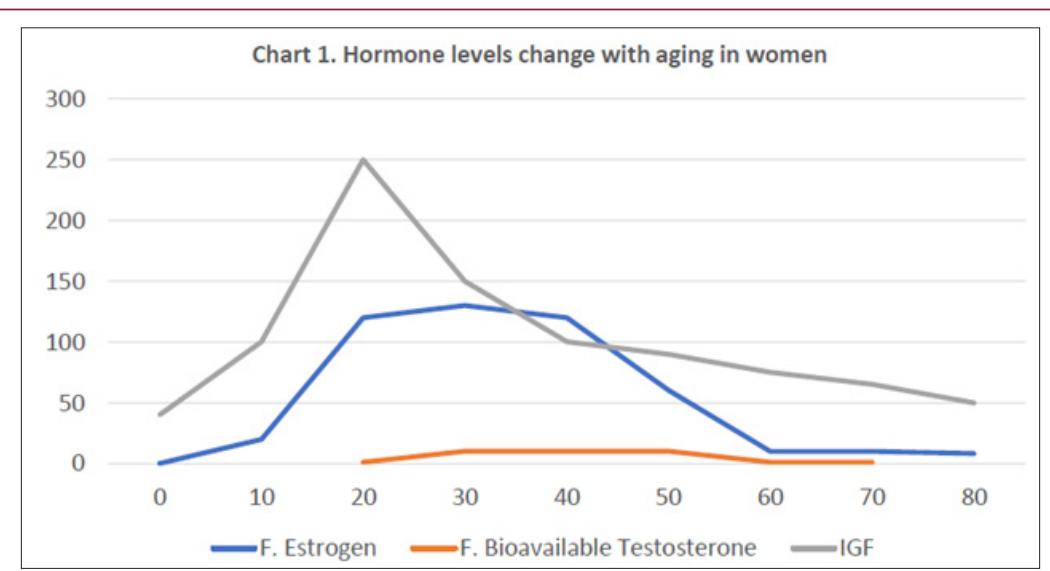

Chart 1: Line chart in color; X-axis: age; Y-axis: relative concentrations of hormones; F: female; IGF: insulin-like growth hormone ${ }^{33}$.

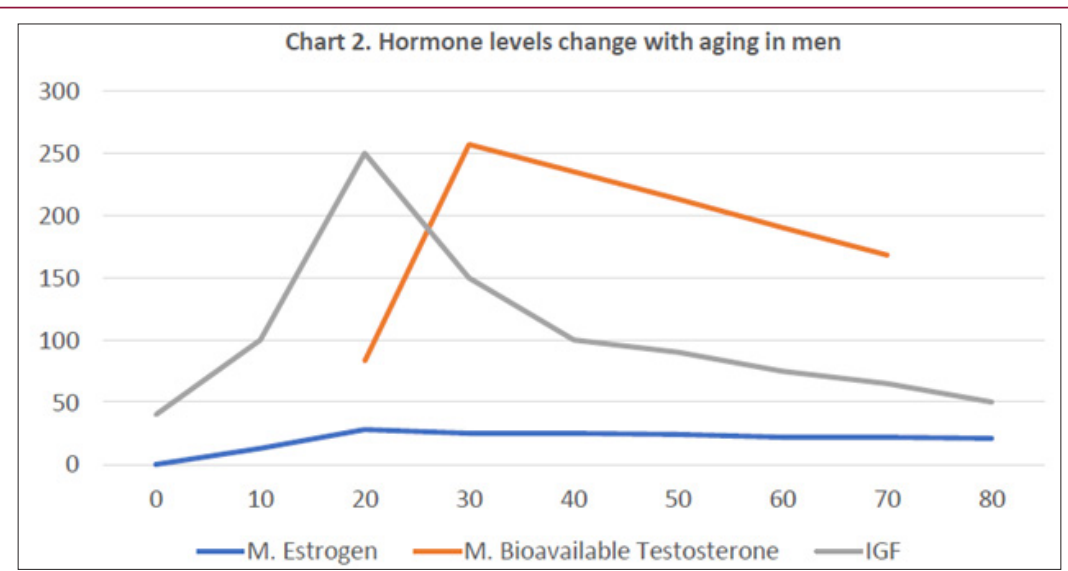

Chart 2: Line chart in color; X-axis: age; Y-axis: relative concentrations of hormones; M: male; IGF: insulin-like growth hormone ${ }^{33}$.

Degradation of Paracrine Molecules: Paracrine molecules spontaneously degrade within few days if the local etiology is eliminated. Theoretically, cytokine antagonists not only inhibit the local inflammation or the angiogenesis but also change the process of osteoclastogenesis.

The Tone Between Radiopacity and Radiolucency (Illustration 2): The abnormal bone remodeling is a result of the imbalance between osteoblastogenesis and osteoclastogenesis. Osteoblasts take a leading role in the maturation of osteoclasts, while the lifespan of osteoclasts is one-sixth of the lifespan of osteoblasts. Therefore, it is possible that osteoclasts are influenced by cytokines becomes the initial event of a local osteogenic lesion. A radiolucency will form if the osteoclastogenesis is accelerated by cytokines. A radiopacity will develop when active osteoclasts lead osteoblasts on its function by the S1P pathway. Osteoblasts' function presents with the deposition of new bone on a pre-existing surface. It is called an appositional growth [1]. This entangling relation allows oral and maxillofacial osteogenic conditions to take on an appearance of a central radiolucency with a peripheral radiopacity or a central radiopacity with a peripheral radiolucency. That is the tone between radiolucency and radiopacity.

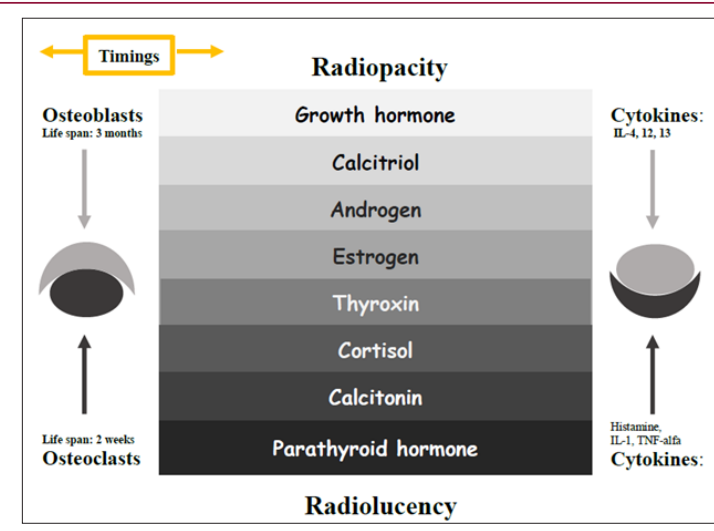

Illustration 2: Triple-hit frame: The tone between radiolucency and radiopacity are modified by molecules from endocrine and paracrine systems (in color). IL: interleukin; TNF: tumor necrosis factor. 


\section{Osteoblastogenesis and Osteoclastogenesis (Illustration 1) and (Table 1)}

For a healthy adult, the osteoblastogenesis maintains stable that is supported by normal levels of GH, Estrogen, Androgen and Calcitriol and is controlled by a negative feedback of Sclerostin. The balance could be broken if the level of female Estrogen drops with age or the level of Calcitriol drops due to the life style. For an adult with hypocalcemia or hypercalcemia, the systemic osteoclastogenesis is triggered by abnormal levels of PTH and Calcitonin. Cytokines are released because of local irritations, such as ischemia, mechanical force, and inflammation; however, some cytokines activate the local osteoclastogenesis and others in the opposite way. The sum outcome relies on the level of the general osteoblastogenesis. The scale tilts to the osteoclastogenesis side if the level of the general osteoblastogenesis is low and to the osteoblastogenesis side if the general osteoblastogenesis is high.

\section{Discussion}

\section{Hypothesis: Triple-Hit Frame (Illustration 3)}

The systemic imbalance of hormones' levels and the local accumulation of paracrine molecules consistently working with an osteogenic multicellular group for 3-6 months are considered non-genetic risks of oral and maxillofacial osteogenic conditions (Illustration 3).

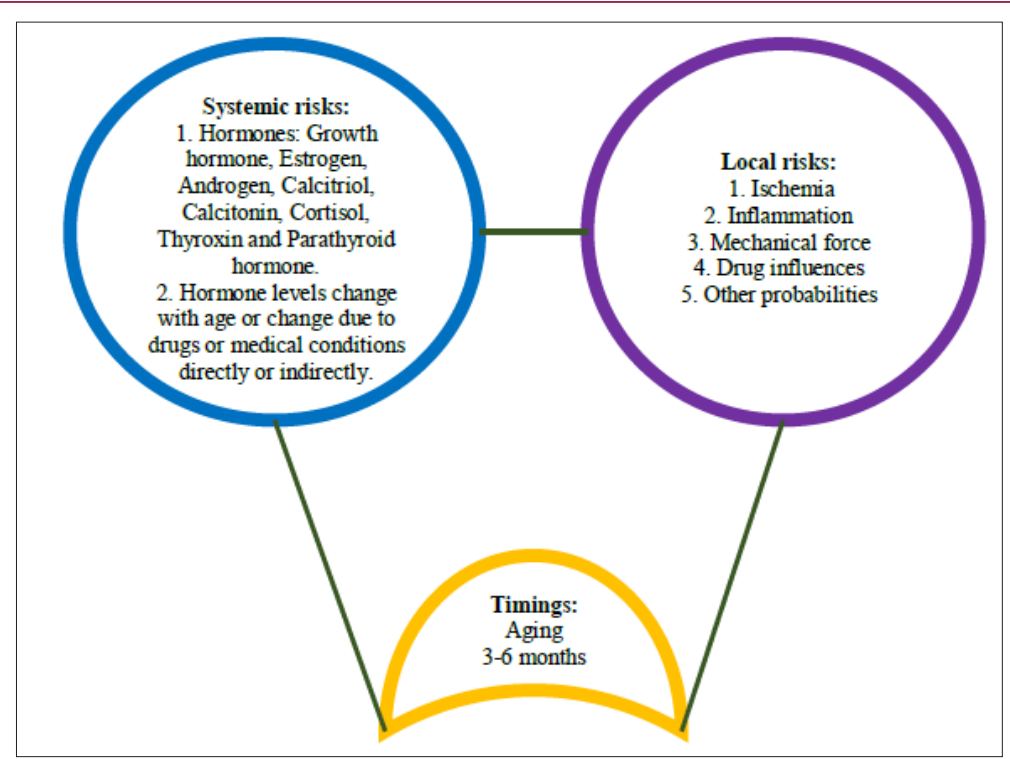

Illustration 3: Triple-hit frame for understanding non-genetic risks of oral and maxillofacial osteogenic conditions: timings, systemic and local risks.

\section{Examples: Two Oral and Maxillofacial Osteogenic Conditions}

Periapical and Focal Cemento-Osseous Dysplasia: They are common in females with a predilection for the third and sixth decades [34]. The common feature is a periapical radiolucency with a peripheral corticated margin. The low level of estrogen maintains a low level of osteoblastogenesis. A routine periapical mechanical force causes cytokines accumulating in apical regions. Osteoclastogenesis takes a priority resulting in a radiolucency formation in the center. The activated osteoclast stimulates osteoblasts on its function by the S1P pathway. The peripheral bone formation occurs.

Cementoblastoma and Osteoblastoma: They are common in adults younger than 30 years old [34]. The common feature is a central radiopacity with a peripheral radiolucent rim. The high level of growth hormone maintains a high level of osteoblastogenesis. A routine periapical mechanical force causes cytokines accumulating in apical regions. Osteoblastogenesis takes a priority resulting in a radiopacity formation in the center. The activated osteoblast stimulates osteoclasts on its function by the RANKL pathway and the M-CSF pathway. The peripheral bone resorption occurs.
All in all, the triple-hit frame (timings, systemic and local risks) provides a tool to explore non-genetic risks of oral and maxillofacial osteogenic conditions. The tone between radiolucency and radiopacity in jaws show some patterns that we can follow.

\section{Acknowledgement}

The author was grateful to Dr. David MacDonald-Jankowski's contribution in many ways towards the completion of the paper.

\section{References}

1. Vinay Kumar, Abul K Abbas, Nelson Fausto, Jon C Aster (2010) Robbins and Cotran Pathologic Basis of Disease. Saunders-Elsevier Inc 26: 12061210.

2. V Shankar Ram, Parthiban, Uma Sudhakar, Nimisha Mithradas, Ramachandra Prabhakar (2015) Bone biomarkers in Periodontal Disease: A Review Article. Journal of Clinical and Diagnostic Research 9(1): ZE07-ZE10.

3. Jianwen Wei, Gerard Karsenty (2015) An overview of the metabolic functions of osteocalcin. Rev Endocr Metab Disord 16(2): 93-98.

4. Yanling Xiao, Jara Palomero, Joanna Grabowska, Liqin Wang, Iris de Rink, et al. (2017) Macrophages and osteoclasts stem from a bipotent progenitor downstream of a macrophage/osteoclast/dendritic cell progenitor. Blood Advance 1(23): 1993-2006. 
5. Susan R Wilson, Christoph Peters, Paul Saftig, Dieter Bromme (2009) Cathepsin K Activity-dependent Regulation of Osteoclast Actin Ring Formation and Bone Resorption. The Journal of Biological Chemistry 284(4): 2584-2592.

6. Jin Hee Park, Na Kyung Lee, Soo Young Lee (2017) Current Understanding of RANK Signaling in Osteoclast Differentiation and Maturation. Molecules and Cells 40(10): 706-713.

7. Johannes Keller, Philip Catala Lehnen, Antje K Huebner, Anke Jeschke, Timo Heckt, et al. (2014) Calcitonin controls bone formation by inhibiting the release of sphingosine 1-phosphate from osteoclasts. Nature Communications 5: 5215.

8. H van Lierop, NAT Hamdy R, L van Bezooijen, CW Lo wik, SE Papapoulos (2012) The Role of Sclerostin in the Pathophysiology of Sclerosing Bone Dysplasias. Clinic Rev Bone Miner Metab 10(2): 108-116.

9. E Mrak, I Villa, M Losa, F Guidobono, A Rubinacci (2007) Growth hormone stimulates osteoprotegerin expression and secretion in human osteoblast-like cells. Journal Endocrinology 192(3): 639-645.

10. Khosla S, Oursler MJ, Monroe DG (2012) Estrogen and the Skeleton. Trends in endocrinology and metabolism: TEM 23(11): 576581.

11. Matthew M Roforth, Elizabeth J Atkinson, Ellis R Levin, Sundeep Khosla, David G Monroe (2014) Dissection of Estrogen Receptor Alpha Signaling Pathways in Osteoblasts Using RNA-Sequencing. PLOS one 9(4): e95987.

12. Mohamad NV, Soelaiman IN, Chin KY (2016) A concise review of testosterone and bone health. Clinical Interventions in Aging 11: 13171324.

13. Stavros C Manolagas, Charles A O'Brien, Maria Almeida (2013) The role of estrogen and androgen receptors in bone health and disease. Nat Rev Endocrinol 9(12): 699-712.

14. Nabanita S Datta, Abdul B Abou-Samra (2009) PTH and PTHrP Signaling in Osteoblasts. Cell Signal 21(8): 1245-1254.

15. Marjolein van Driel, Johannes PTM van Leeuwen (2014) Vitamin D endocrine system and osteoblasts. BoneKey Reports 3: 493.

16. Naoyuki Takahashi, Nobuyuki Udagawa, Tatsuo Suda (2014) Vitamin D endocrine system and osteoclasts. BoneKey Reports 3: 495.

17. Susame Granholm, Pernilla Lundberg, Ulf H Lerner (2007) Calcitonin inhibits osteoclast formation in mouse hematopoietic cells independently of transcriptional regulation by receptor activator of NF-kB and c-Fms. Journal of Endocrinology 195(3): 415-427.

18. Graham R Williams (2013) Thyroid Hormone Actions in Cartilage and Bone. European Thyroid Journal 2(1): 3-13.

19. Gordon L Klein (2015) The effect of glucocorticoids on bone and muscle. Osteoporosis and Sarcopenia 1(1): 39-45.

20. James J Logie, Sadaf Ali, Kathryn M Marshall, Margarete MS Heck, Brian R Walker, et al. (2010) Glucocorticoid-Mediated Inhibition of Angiogenic Changes in Human Endothelial Cells Is Not Caused by Reductions in Cell Proliferation or Migration. PLOS one 5(12): e14476.

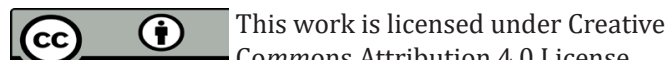

Submission Link: https://biomedres.us/submit-manuscript.php
21. Masako Yoshimatsu, Hideki Kitaura, Yuji Fujimura, Haruka Kohara, Yukiko Morita, et al. (2015) IL-12 Inhibits Lipopolysaccharide Stimulated Osteoclastogenesis in Mice. Journal of Immunology Research p. 1-10.

22. Yamada A, Takami M, Kawawa T (2007) Interleukin-4 inhibition of osteoclast differentiation is stronger than that of interleukin-13 and they are equivalent for induction of osteoprotegerin production from osteoblasts. Immunology 120(4): 573-579.

23. Tobias Braun, Jochen Zwerina (2011) Positive regulators of osteoclastogenesis and bone resorption in rheumatoid arthritis. Braun and Zwerina Arthritis Research \& Therapy 13(4): 235.

24. Martin Biosse Duplan, Brigitte Baroukh, Michel Dy, Marie Christine de Vernejoul (2009) Histamine Promotes Osteoclastogenesis through the Differential Expression of Histamine Receptors on Osteoclasts and Osteoblasts. Am J Pathol 174(4): 1426-1434.

25. JM Kanczler, ROC Oreffo (2008) Osteogenesis and angiogenesis: the potential for engineering Bone. European Cells and Materials 15: 100114.

26. Masabumi Shibuya (2011) Vascular Endothelial Growth Factor (VEGF) and Its Receptor (VEGFR) Signaling in Angiogenesis: A Crucial Target for Anti- and Pro-Angiogenic Therapies. Genes \& Cancer 2(12): 1097-1105.

27. Hideki Kitaura, Keisuke Kimura, Masahiko Ishida, Haruki Sugisawa (2014) Effect of Cytokines on Osteoclast Formation and Bone Resorption during Mechanical Force Loading of the Periodontal Membrane. The Scientific World Journal 2014: 617032.

28. Robling AG, Niziolek PJ, Baldridge LA, Condon KW (2008) Mechanical stimulation of bone in vivo reduces osteocyte expression of Sost/ sclerostin. The Journal of Biological Chemistry 283(9): 5866-5875.

29. I Ning E Wang, Jing Shan, Rene Choi, Seongcheol Oh (2007) Role of Osteoblast-Fibroblast Interactions in the Formation of the Ligamentto-Bone Interface. Journal of Orthopaedic Research 25(12): 1609-1620.

30. Kenta Yamamoto, Tsunao Kishida, Yoshiki Sato, Keisuke Nishioka (2015) Direct conversion of human fibroblasts into functional osteoblasts by defined factors. PNAS 112(19): 6152-6157.

31. Nahum Rosenberg, Orit Rosenberg, Michael Soudry (2012) Osteoblasts in Bone Physiology-Mini Review. Rambam Maimonides Medical Journal, Clinical Implications of Basic Research 3(2): e0013.

32. Naveen Aggarwal, Salman Razvi (2013) Thyroid and Aging or the Aging Thyroid? An Evidence-Based Analysis of the Literature. Journal of Thyroid Research p. 1-8.

33. Riia K Junnila, Edward O List, Darlene E Berryman, John W Murrey, John J Kopchick (2013) The GH/IGF-1 axis in aging and longevity. Nat Rev Endocrinol 9(6): 366-376.

34. Brad W Neville, Douglas D Damn, Carl M Allen, Jerry E Bouquot (2009) Oral and Maxillofacial Pathology. Saunders-Elsevier Inc 14: 640-656.

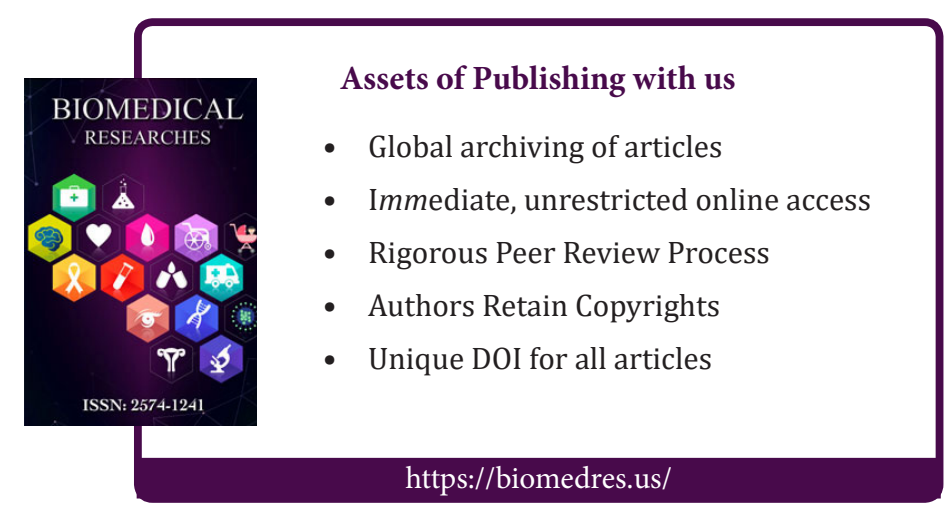

